\title{
Advanced Analytics in Process Industries
}

The full power of advanced analytics requires not only acquiring new technology and analytics solutions, but also helping people improve their expertise and adopt new ways of working.

The Internet of Things (IoT), industry 4.0, advanced analytics, digital technologies, and big data have all generated enormous hype for their potential to transform every facet of business. To date, the dialogue around these tools has focused primarily on consumer-facing industries, such as retail and financial services; on large industrial companies, such as GE; and on leading digital players, including Amazon, Google, and Netflix. Beyond the spotlight, however, manufacturers in process industries - also commonly referred to as heavy or capital-intensive manufacturing-have been early to adopt many of the latest advances. For decades the sector has not only generated large amounts of data but also combined science, engineering knowledge, classical statistics, and powerful modeling into advanced-process-control (APC) systems that run key assets efficiently.

\section{The processes of heavy manufacturing}

Heavy manufacturing broadly uses two types of processes. In structured, deterministic ones, wellestablished thermodynamic, kinetic, and engineering principles enable the manufacturer to use available data to predict the results of the process with a high degree of confidence. For example, a distillation column separates crude oil into multiple compounds with different boiling points - an inherent chemical property that informs the design of the column and allows the manufacturer to determine an optimal output based on a relatively small number of variables. By contrast, less structured, nondeterministic processes are a bit messier: the problem's scientific and engineering definition isn't fully known and data are not available. In mining, a chemical reactor that pro- cesses mineral mix involves a range of unknowns because the exact combination of minerals is not knowable. Accordingly, the process is less controllable, and efforts to improve performance are far more complex.

Since APC systems are expensive to develop and historically have been effective only for well-understood, less-complex processes, APC usage has generally been limited to the largest, most critical ones. Smaller, as well as secondary and morecomplex processes have been left without suitable process controls (see sidebar, "The processes of heavy manufacturing"). And while process industries generate huge volumes of data, their process-management and information-technology capabilities are not as advanced as those of other industries. As a result, thus far manufacturers have lagged behind in systematically deploying data analytics to extract the substantial value hidden in the insights they contain.

The good news is that over the past several decades, entirely new and more affordable manufacturing analytics methods and solutions have emerged, and they are now reaching market maturity as part of Industry 4.0. These solutionswhich provide easier access to data from multiple data sources, along with advanced modelling algorithms and easy-to-use visualization approaches - could finally give manufacturers new ways to control and optimize all processes throughout their entire operations.

But new technology is just one part of the equation. To achieve a strong financial impact from improvements in analytics, manufacturers must also consider the human factor. As in previous efforts to optimize production, such as lean manufacturing or ISO quality standards, change-management capabilities will be crucial. The new horizon in analytics will achieve its full impact only when 
manufacturers enhance skills across the entire organizations so that the new methods and solutions become a part of the day-to-day routine.

Analytics can improve performance tremendously while reducing costs: the value, spread over thousands of opportunities, can be worth tens or even hundreds of millions of euros across a company's site network. The key challenge is deploying analytics hundreds or thousands of times to achieve the desired outcomes. Manufacturers must therefore embark on an analytics-transformation effort that reaches all the way from the shop-floor operators (who steer processes) to process engineers (who use deep insights that will drive the next wave of improvements) to managers (who constantly oversee performance).

This course requires manufacturers to develop the management capabilities and mind-sets that can mobilize the entire organization to harness the new analytics technology. Heavy manufacturers that apply this approach consistently-process by process, plant by plant, and location by location around the world-will capture the full value of the new optimization opportunities and more value from their physical assets and people.

Would you like to learn more about our Operations Practice?

The evolution of automation technology in heavy manufacturing

In heavy industry, current process-control systems can run, say, entire chemical plants from a control room in fully automated mode, with operations visualized on computer screens. Data steer processes through proportional-integral-derivative (PID) algorithms that manage local loops.

Certain industries, such as oil and gas refining, have taken the process-control logic a step further by using APC systems to run continuous-optimization models. Indeed, for the past decade, the heavy-manufacturing sector has been increasing its profits per hour by successfully applying APC technologies in many different processes. These initial models, which require a static, well-described (or "deterministic") problem, are extremely powerful. Their main drawback, and the reason they have been implemented only in specific settings, is the expense: APC systems can cost $€ 0.5$ million for a distillation column or $€ 1.5$ million for a hot strip-mill furnace. Consequently, the return on investment (ROI) typically makes sense only in big processes.

Next-generation analytics, optimization infrastructure, and tools

But heavy industry is now on the brink of the digital revolution. IoT technology-which includes affordable sensors, reliable data transmission, and powerful control software - can generate and track information from every machine and step in a manufacturing process. Similarly, affordable storage options (both high-performance databases and cloud offerings) have made it possible to aggregate and manage vast amounts of data. Last, modular, easy-to-use analytical packages, especially those that also include artificial intelligence and machine learning, can harness and process this information to provide new levels of insight. In addition, many algorithms and software tools are being commoditized, making them easier for employees to use without significant training.

Conventional APC systems have monitored and managed large, deterministic processes. Thanks to the aforementioned technological advances, manufacturers can now deploy new, low-cost control and optimization solutions across midsize deterministic processes and midsize-to-large nondeterministic processes, which until now haven't benefited from this kind of sophisticated technology An evaluation of the total value of potential performance improvements illuminates the enormous opportunity for manufacturers that get it right. As an example, a large multinational company typically has a couple of dozen big processes (such as a catalyst-inhibitor addition in a chemical process) that if optimized could cut costs or generate productivity improvements of $€ 1$ million each. However, manufacturers also have hundreds to thousands of midsize opportunities, such as steam-superheat controls (the benefits range up to $€ 50,000$ per boiler a year) or undersized valve-restricting cooling (up to $€ 70,000$ a year). A chemical company with 150 sites, for example, and 10 
to 100 processes to optimize could have 1,500 to 15,000 opportunities whose recurring annual cost savings could range from $€ 50$ million to $€ 500$ million.

By using analytics systematically, manufacturers unlock savings from three sources, each delivering an equal portion of the value. First, analytics gives process engineers greater visibility into every process, which they can therefore steer more effectively. Second, it supports better management-for example, by helping operators to spot when a process deviates from defined key performance indicators (KPIs). This visibility allows them to detect movements and take corrective action much more quickly. Third, it uncovers hidden process restrictions that can often be resolved with small capex investments. Besides making employees more efficient, it can help to remove critical human bottlenecks, freeing up time and resources that can be directed to additional improvements in plants.

Foundational elements of analytics-driven process optimization

Manufacturers face a key challenge in analytics: deploying it hundreds of times while assuring consistency, quality, and the development of a continuous-improvement cycle that supports further advances. External parties can assist in setting up the framework, delivering the first successful pilot implementation, designing the organizational processes, and building up the capabilities of early users. Eventually, however, manufacturers will have to implement sustainable practices and install experts across the global organization, much as they do for quality assurance, energy management, hazards and safety, compliance, and other functional practices.

Four expertise areas are crucial in setting up the right organizational support so that the company can not only aggregate and analyze data, but also act on the findings.

IT expertise. This category includes employees with the knowledge and skills to aggregate data from sensors (including their location, type, and accuracy) and to store this information in vari- ous platforms (the cloud, on-site servers, off-site servers, the external cloud). IT also deploys and maintains the new systems, sensors, and software solutions.

Domain expertise. Data analytics, by itself, can't deliver the full range of impact and value without an understanding of how processes behave and how local plants are set up. Frequent interactions with local experts are essential to construct a useful optimization model. Process engineers can collaborate with data-analytics specialists to base models of outcomes (such as yield and energy use) on defined inputs and can use expert knowledge to solve problems throughout the process. Moreover, they can update value-driver trees and develop a solution database for future reference.

Advanced analytics. Data must also be structured by time, batch, combination of time, and batch and time delay, as well as cleaned to identify and visualize outliers and missing data. Analytics tools, such as value-driver trees, can help determine the critical parameters and cluster data by tag information - and they are flexible to reprogram. Employees - in particular, process engineers and operators-must be trained in the new technology and be able to work with it day by day and to act correctly on its recommendations.

Change management. Transformation professionals define improvement measures, build optimizers for operators, and track performance and KPIs. Implementation efforts use dashboards and apps to visualize performance in real time, and automation embeds improvement measures in APC software. More often than not, surprising additional findings will emerge to challenge existing knowledge and dispositions. More real-time information ultimately changes how decisions are made and leads to an agile way of operating.

Methodically progressing through the organization - process by process, opportunity by opportunity-will ensure both consistency and maximum impact.

Driving an analytics transformation across a global organization

A successful analytics transformation requires 
manufacturers to refine a single process and then develop the capabilities to replicate that approach to optimization hundreds or thousands of times across the enterprise. In our experience, manufacturers typically already have the domain and IT expertise to capture and store massive amounts of data. To ignite and sustain an analytics transformation, executives should focus on strengthening organizational capabilities in advanced analytics and change management.

Piloting the analytics approach

An analytics transformation starts by identifying specific processes to serve as pilots. Ideally, these initial projects can demonstrate the viability and value of analytics and realize improvements quickly.

The next step is to implement a learning and improvement process. A good part of optimization involves using analytics tools to gain a better understanding of the drivers of greater efficiency and cost savings. Normally, analytics highlights opportunities for small capex investments to improve the entire system's performance. Lessons from these initial efforts can be integrated into a longer-term one to develop a more permanent solution.

Capturing value is first and foremost about people-from selecting the right process for collecting data to carrying out the analytics to implementing the resulting insights on the shop floor. Capturing that value is also about infusing analytics throughout the organization and building the capabilities to set up more effective processes. Manufacturers may be tempted to leapfrog the immediate, incremental approach in an effort to seize the larger prize more quickly. Others can get stuck at an early stage by taking too long to design and implement a customized technology solution. In our experience, it does not make sense to spend two years and several million euros on the optimal approach if inexpensive data lakes and analytics can help organizations to start delivering value within the first few months.

Heavy manufacturers and their staffs must also embrace a new mind-set - one centered on data- driven decision making and on the processes to gather, analyze, and apply these insights to draw the right conclusions.

The age of analytics: Competing in a data-driven world

Almost all the classical transformation-success factors are required for an analytics transformation: setting clear aspirations, getting focus and buy-in from top management, engaging employees through clear and well-executed communication initiatives, identifying skill gaps, planning to build the required capabilities, defining pivotal roles, and committing sufficient resources.

Given the large size of the individual opportunities and the complexity of interacting with several departments and business units, setting up a new program-management office is critical to a successful analytics transformation. Once the pilot has been completed, the change-management team should identify opportunities, prioritize locations, formulate the communication plan, set up the required IT support, and select and train experts on the job in successive waves. The preparatory phase includes training in software tools. Experienced engineers should be ready to teach and help other employees and to update training materials by drawing on lessons from early waves of training.

This approach uses a learn-and-transform model: groups of engineers follow a classic transformation journey (prepare, diagnose, design, plan, implement, and sustain) that's broken down into three- to four-week sprints. Each sprint includes joint learning, on-site application, and feedback sessions with trainers to improve retention. In our experience, a small group of experts can administer training to 200 engineers within 18 months, achieve an impact within four to five months, and develop the rollout plan for a site in parallel. In this way, an organization can develop enough process engineers to cover 150 sites in just 18 months. It's critical, in the journey, not only to capture value from specific opportunities but also to have engineers develop a road map of improvements for the next few years. 